\title{
Correction to: A Simple Method of the Haulage Cycles Detection for LHD Machine
}

Wioletta Koperska (1), Artur Skoczylas (1), and Paweł Stefaniak (1)

\section{Correction to: \\ Chapter "A Simple Method of the Haulage Cycles Detection for LHD Machine" in: M. Hernes et al. (Eds.): \\ Advances in Computational Collective Intelligence, CCIS 1287, https://doi.org/10.1007/978-3-030-63119-2_27}

In the originally published version of the chapter 27 , the first names and surnames of the authors were used in an incorrect order. This has been corrected. 